\title{
Equitable Mindfulness: A Framework for Transformative Conversations in Higher Education
}

\author{
Tiara A. Cash, MS \\ Nika Gueci, EdD, MA \\ Teri Pipe, PhD, RN \\ Center for Mindfulness, Compassion, and Resilience - Arizona State University
}

\begin{abstract}
Background: Mindfulness, the practice of present moment experience, can be employed as a tool for grounding in difficult conversations and creating pathways for transformative change in communities.

Aim: As educators continue to teach the practices of mindfulness in primary, secondary, and postsecondary institutions to promote personal and academic wellbeing, it is important to recognize the inherent power of mindfulness practice to stimulate actions against oppressive systems, instead of continuing to support them by using curriculum focused on desensitizing individuals to that system. The outlined framework aims to create a platform for the promotion of sustained action.

Methods: This article will outline the framework and model of Equitable Mindfulness used within curricula to create containers, hold space, and begin difficult conversations about creating and sustaining diverse and inclusive spaces and strategies.

Results: Rooted in the foundations of mindfulness, the Equitable Mindfulness framework was created to promote personal and societal introspection while bridging gaps between communities and breaking down barriers against inclusive practices.
\end{abstract}

Conclusions: Researchers are continuing to collect mixed-methods data on how Equitable Mindfulness is perceived, it's validity in the field, and its relationship to mediating difficult topics.

Submitted 3 August 2020: accepted 23 November 2020

Keywords: Mindfulness, Community Building, Well-Being, Anti-Oppressive Conversations and Systems

Mindfulness is a term and a practice that is used considerably these days in entertainment circles, athletics, healthcare, and relevant to this paper, higher education. Mindfulness is often represented as a tool to facilitate leadership and success, but mindfulness holds great potential to serve all people through community engagement and inclusive practices.

Mindfulness, as defined by Jon Kabat-Zinn, is a "non-judgmental, moment-to-moment awareness" (Kabat-Zinn, 1990 , p. 2). As a practice, mindfulness can improve individual well-being and provide personal benefits, such as improved time management skills (Kabat-Zinn, 1990); a more balanced mood and equanimity (Rogers, 2016); decreased anxiety (Hall, 2009); increased self-efficacy (Bohecker \& Doughty Horn, 2016; Pipe, Bortz, Dueck et al., 2009); compassion and kindness toward self and others (Pipe \& Bortz, 2009; Pipe, Bortz, Dueck et al., 2009); and reduction of burnout and increased resilience (Montero-Marin et al., 2015). In research contexts, mindfulness is most frequently taught at the individual level, or within the social context of a small group or class. 
Mindfulness has been studied and practiced in the west primarily through two distinct backgrounds: (1) rooted in religious traditions from India and East Asia (Olendzki, 2005) and (2) in clinical practices and trainings, such as mindfulness-based stress reduction (MBSR; Kabat-Zinn, 1990). The former is anchored in (or stems from) Zen, Vipassana or Tibetan techniques of Buddhism, and religious ideologies of Hinduism (Fulton \& Siegel, 2013; Grabovac, Lau, \& Willet, 2011; Krygier et.al., 2013; Robins, 2002). The latter, as seen in popular culture, focuses primarily on the health benefits of mindfulness. These clinical western secular spaces emphasize stress relief and the physical and psychological benefits that mindfulness practices can evoke, such as decreased anxiety and stress, and increased resilience (Carmody \& Baer, 2008; Grossman, Niemann, Schmidt, \& Walach, 2004; Kabat-Zinn, 1990; Hawley et. al., 2014).

The secular path to mindfulness is seen a point of entry for skeptics of the religious aspects of mindfulness and those who study hard sciences. Research sample populations, however, are usually White/Western, Educated, Industrialized, Rich, and Democratic (WEIRD), i.e., not representative of the global majority (Henrich, J., Heine, S., \& Norenzayan, A., 2010). Research that relies on WEIRD populations is exclusionary; its findings cannot and should not be generalized to all populations, nor can they produce actionable conclusions. For example, time constraints, financial limitations, differences in religious ideologies, and cultural preferences of other stress reduction techniques have been suggested as barriers to mindfulness practice (Woods-Giscombé \& Gaylord, 2014; García-Campayo, Demarzo, Shonin, \& Van Gordon, 2017). These suggestions are socially defined as reasons for "vulnerable" and "minority" populations' lack of engagement in these data-driven secular mindfulness training programs. Mindfulness, however, has also been cited and described as a universal practice, inherent in the fabric of human existence (Olendezki, 2005; Forbes, 2016). With this context, research and practice of mindfulness requires a broader lens than the current frame of western secular philosophy.

\section{MINDFULNESS, SOCIAL JUSTICE, AND EQUITY}

Research in the intersection of mindfulness and social justice (i.e., the practice of mindfulness for social change) is novel compared to the research on mindfulness for health and wellness. Mindfulness for social justice in social work and the justice system (specifically for lawyers) have received the most attention (Gonzalez, 2017; Harris, 2011; Hick \& Furlotte, 2019; Wong, 2004). Recently, mindfulness and social justice began emerging as an area of study and practice in western educational systems, independently, as a form of pedagogy and practitioner work for those who are administering anti-oppressive curricula in higher education.

This shift from individual well-being to a broader societal perspective conceptualizes mindfulness as both antioppressive and antiracist (Cannon, 2016; Berila, 2015). In this way, mindfulness is a route to social justice because of its ability to support the individual in engaging in self-awareness, introspection, community engagement, compassion, and resilience (Magee, 2016).

The sub-field of mindfulness and social justice focuses on translating western secular mindfulness to mindfulness for the larger community. As stated by Forbes (2016), mindfulness as related to social justice is a "demand for universal justice with critical theory that challenges the status quo of power and with a call for our highest personal development." He follows this statement with the description of "McMindfulness," which practitioners use to describe mindfulness that does not promote natural compassion and universal recognition of our embodied connections within this practice. Rather, McMindfulness focuses on egotistical comfort and adjustment to life for private gain. Without critically examining the social context, “...mindfulness as a practice and discourse focused on 
the self minimizes social critique and change and contributes to keeping existing social injustices and inequitable power structures intact" (Forbes, 2016).

This distinction is important in forging a path for Equitable Mindfulness to be leveraged to promote universal inclusiveness. By framing the need for inclusive speech, education, and research within the field of secular mindfulness, higher education can formally explore and explicate the practices' capabilities in curriculum development and implementation. Equitable Mindfulness stems from the philosophical underpinnings that the practice is truly for all people, rather than a select few. Understanding that mindfulness at its core can be universal, community-based, and inherently inclusive can provide a more complete picture of mindfulness and its benefits as a practice and in research. This lens can create space for the use of mindfulness to curate and support inclusive and brave spaces for intentional engagement in difficult conversations, i.e., inclusive, anti-oppressive curriculum.

\section{EQUITABLE MINDFULNESS FRAMEWORK}

Mindfulness can be defined as awareness, cultivated by paying attention in a sustained and particular way, i.e., on purpose, in the present moment, and non-judgmentally (Kabat-Zinn, 2012). Cited in a wealth of research studies over the past few decades, the practice of mindfulness is not novel or selective, and it was not created by the medical community or western society. Mindfulness practice is an ancient and deeply rooted practice that stems from most cultures and spiritual traditions. According to Olendzki (2005), "The practice of attending carefully to the details of one's own present experience is probably as old as humankind itself." Mindfulness is mentioned in cultures spanning the globe, including but not limited to cultures in East and South Asia, Africa, the African diaspora, ancient and current Indigenous cultures around the world, and ancient Europe (Hanson, McLeod, \& Ross, 2017; Le \& Shim 2014; Nussbaum, 2013; Phillips, 1990). However, mindfulness researchers rarely acknowledge its roots.

Mindfulness, as an experience and practice, is universal, but calling all mindfulness practices by one name and viewing them through one lens obscures the universal ideology that saturates the nuances of each culture's practice. Further, by ignoring the social inclusion and regard for humanity at the core of most mindfulness practices, we overlook the power of these tools for enhancing human experience and healing (Forbes, 2016).

Equitable Mindfulness grew out of the need to recognize the multi-cultural and universal experience of mindfulness and protect against mindfulness becoming understood as an exclusive practice to enhance success. Equitable Mindfulness is integral in creating shared spaces (extending an invitation of an individual's mindfulness practice to another person) and brave containers (spaces of conversation and community initiated by the facilitator and continued and managed by the participants). Equitable Mindfulness can help practitioners, professors, and teachers facilitate difficult conversations and practices of inclusion and equity in higher education by encouraging practitioners to acknowledge and engage with an individual's personal and cultural nuances. The importance of this work has never been greater than in the current context, as emotions stemming from hurt, anger, isolation, pain, political polarization, and a need for human connection and healing are salient.

\section{INCEPTION OF EQUITABLE MINDFULNESS WITHIN THE LOCAL CONTEXT}

Next, we describe the local context in which Equitable Mindfulness was created and implemented. The Center for Mindfulness, Compassion, and Resilience (Center) is housed in a university in the Southwest U.S. with approximately 111,000 students (in-person and online; Arizona State University fall enrollment numbers, n.d.). The institutional 
mission and values are demonstrated in the charter, noting that the university measures itself "not by whom it excludes, but by whom it includes and how they succeed" (Arizona State University Charter, n.d.). The student population of this institution is intentionally diverse, reflecting the population demographics of the state in which it is located. The Center was founded in 2017, and it reflects the institution's values to enhance the well-being of all students, faculty, staff, and community (http://mindfulnesscenter.asu.edu) (Mindfulness comes to ASU, n.d.).

Each year, the Center hosts a conference based on themes relevant to the current social and cultural contexts. In 2019, the Center hosted "Water and Stone: The Power of Mindfulness for Social Change," a two-day curated discussion engaging the community in an exploration of the intersection of mindfulness and social change. To help further the Center's initiative of equity within mindfulness and engaging in social transformation, the Center conducted a mixed methods study, recruiting 55 participants to complete a closed-ended questionnaire and a 20-30 minute interview. The preliminary results indicated that mindfulness is inherently equitable, but equitable access to mindfulness is lacking; and mindfulness increases the awareness of issues and gaps, which may help identify areas in need of change (Bautista et.al., 2019).

The personnel from the Center began creating a curriculum, based on research results, aimed at increasing accessibility to mindfulness and promoting recognition and awareness of social areas in need of transformation (Goodman, 2019). Workshops and presentations began to incorporate the Equitable Mindfulness model, and themed gatherings with mindfulness practices as a foundation were created to promote conversations around difficult concepts among diverse participants. These workshops and presentations were free and open to the community and themes included Diversity in the Workplace, The True Face of Healing, and Othering and Belonging in Education. Participant testimonials from these gatherings are included below.

"These Equitable Mindfulness gatherings have been such a powerful source of insight, growth, and togetherness. I look forward to these gatherings, as each one invites participants to dive deeply into conservations that society at large tends to forgo. The space created is one where both introspection and community connection are alive. The topics are challenging but needed, for the purpose of growth. I am grateful for all I have learned and experienced about myself and my community through these gatherings."

"I have been to several Equitable Mindfulness sessions on diverse topics such as trauma, general mindfulness, and othering and belonging. I've enjoyed the rituals at the beginning, the grounding work that happens, the creation of a safe space through verbal agreement of conversation boundaries, the way silence and discussion are embraced equally, the non-judgmental and welcoming atmosphere (sitting in a circle, coffee available, friendly hellos and goodbyes), and the way delicate topics find their ways into our individual and group healing."

“The Equitable Mindfulness workshops were a beautiful space to feel safe sharing our stories and giving us greater understanding of each person's unique experience and life perspective. It was a wonderful growth experience."

The attraction to the concept of mindfulness as a space of equity, connection, and bridging across demographics sparked the official construction of the Equitable Mindfulness framework; created to assist teachers, practitioners, 
professors, and healthcare workers in creating shared spaces and brave containers for difficult social, cultural, and transformative concepts.

\section{DEFINITIONS IMPORTANT TO EQUITABLE MINDFULNESS}

The following definitions are included to explain the Equitable Mindfulness framework and model.

Mindfulness: Awareness, cultivated by paying attention in a sustained and particular way: on purpose, in the present moment, and non-judgmentally. (Kabat-Zinn, 2012)

Equitable Mindfulness: Presenting the concept of mindfulness to everyone, while being active in our respective communities by removing personal and systematic barriers that work against inclusivity and transformative change. (Cash, n.d.; Kamenetz, \& Knight, 2020)

Holding space: Inviting an individual into one's mindfulness practice by seeing, hearing, and holding what they need to express by using mindful listening and heartfelt compassion without giving advice or attempting to solve problems.

Shared spaces: Each participant in a group intentionally holds space at the same time, co-creating a shared experience-space.

Bridging: Relating to other groups based on deep listening, empathetic space, and recognition of suffering. Rejects that the concept of "them" while recognizing unique differences. A strategic or intentional rejection of othering (the act of making someone feel as if they are not a part of a group; Hass Institute, 2019)

Brave container: An action initiated by the facilitator and continued and managed by the participants to intentionally create a space of conversation and community. Grounded in principles of mindfulness and bridging. This is a departure from the traditional role of the facilitator and the associated power gradient; this container disrupts the hierarchical norm.

\section{POSITED MODEL AND IMPLEMENTATION WITHIN CURRICULUM}

Equitable Mindfulness supports the intersections between mindfulness and social transformation, community activation, social justice, and equity. It is grounded in creating a more equitable world through sustained practice of compassion and mindfulness.

Equitable Mindfulness is built on three tiers: (1) personal protection; (2) mindful activation and sustainability of practice; and (3) creating programming with the demographic in mind. Each tier aligns with one level in the Expansive Model of Mindfulness, created by Gueci and Cash (2020) to describe the broad capacity of mindfulness beyond selfinteraction. As previously mentioned, seeing the expansive capacity of mindfulness beyond the self is integral to understanding how to create inclusive spaces through this work. According to the model, personal protection falls within mindfulness for the self, mindful activation within mindfulness for others, and creating programming with the demographic in mind within mindfulness for community (see references). These placements are important to the deeper understanding of how Equitable Mindfulness itself can also be considered expansive.

Personal protection is experiencing the dedicated practice as a first line defense to encounters, conversations, or situations that might have negative effects on an individual's mental, emotional, or physical state. This tier responds to questions such as, "How do we move through the world intentionally responding instead of automatically reacting?" and "How can we live more authentically through our own values when faced with systematic, societal, or 
cultural malice?"

Mindful activation for systematic change and sustainability of practice explores activating the practices of mindfulness to create transformational change within communities. This tier relies on a dedicated personal practice for sustainability of transformational actions that might be needed for the longevity of social change. This tier focuses on questions such as, "How do we use our personal practice to begin to change the systems in our world that keep us (as a community) from seeing the need for equity and that keep us from creating inclusive environments?” and "What can we do to activate our practices in movements within the community to help ourselves and other allies continue their important work?"

Finally, programming with the population in mind operates on the idea that mindfulness can be inherently inclusive, if the parties that are facilitating understand the inclusive roots of the practice and see mindfulness practice as a skillset that is universal within common humanity. Mindfulness is a learned skill set that is free, no monetary resources are needed to live a life in the present moment with intention. The practices are rooted in what we already have and know about ourselves. For example, we all have breath, and we can all use that breath to anchor in the present. Mindfulness is a way of tapping into the self that has been used for centuries and in numerous cultures and traditions around the world. Understanding these points, we provide a framework for dialogue around the following questions: "How can the community of mindfulness practitioners within the field of secular mindfulness use the Equitable Mindfulness concepts to support inclusive curriculum?" and "What instruction is needed using the Equitable Mindfulness framework to engage workers in the fields of health equity, diversity inclusion and equity (DIE), health promotion/public health, education, healthcare, business, and other emerging sectors?"

The Equitable Mindfulness model (Figure 1) has been explored by the Center within a variety of contexts, including: primary, secondary, and higher education; corporations serving underserved and at-risk populations; athletic departments; and health care students/departments (nursing, medical, health promotion, etc.). The Equitable Mindfulness model's main purpose is to use the already inclusive foundations of mindfulness as a paradigm to invite participants of presentations, workshops, and events into inclusive and brave spaces to engage with the universal premise of mindfulness in conjunction with existing curricula.

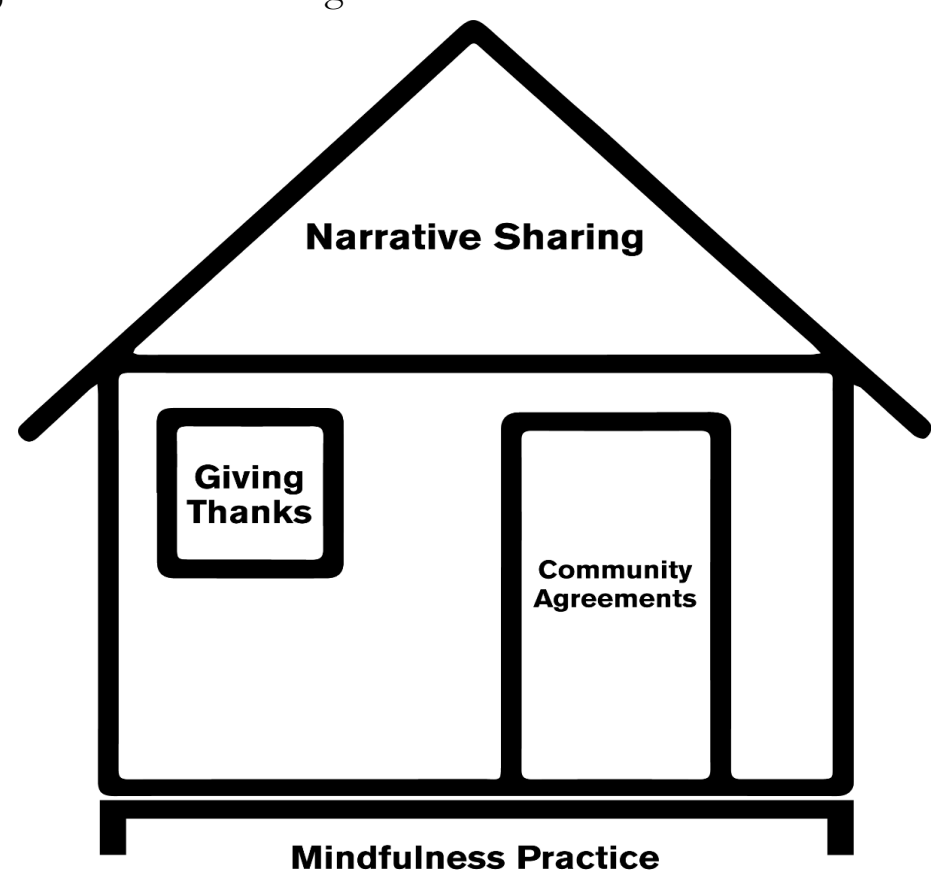

Figure 1. Equitable Mindfulness Model 
Presented in sequential steps, the Equitable Mindfulness model starts at the beginning of the event, class, workshop, or training, before engaging with the field-specific topics. The model components include beginning the session with a mindfulness practice, sharing community agreements and gathering consent, giving thanks to communities that have importance to the topic but who are frequently not acknowledged, and laying groundwork for community interaction by sharing a story (narrative). Although these steps should be taken in sequence, the information that the practitioner or facilitator includes in each step will depend on the population that is being served and who is engaging with the curriculum. As an example, the indigenous acknowledgement in Arizona will be different than the indigenous acknowledgement of communities in South Africa, due to the differences in original people. It is also important to note that facilitators set up all workshop materials before participants arrive (mats, papers, journals, chairs, and tables). Facilitators have found that when participants arrive in a space that is already organized, the thought and preparation contribute to a sense of security.

Each step for the Equitable Mindfulness model is represented by a part of a house (home). In many cultures, the home is a symbol of respite, shared space with family and friends, and a secured and sheltered container. The authors acknowledge that in some cases the home may not be a space of safety, and in those cases, the model can be depicted using any building that is relatable to the participants.

\section{Beginning the Session/Class/Event with a Mindfulness Practice: Foundation}

This experience is meant to set the "foundation" for the rest of the time spent together. Having an opening practice at the beginning of the event assists in creating both a physical calming response (stimulation of the vagus nerve) and allows participants time to disengage from experiences they had prior to arriving and settle into the moment. This step is depicted as the foundation of the home because it is integral in setting the structure of comradery. This experience should be expansive beyond the initial self to others, community, and extended to broader humanity (Gueci \& Cash, 2020). Just as with the foundation of a home, although not always seen, this component lays the groundwork for the expansive shared space that the container is built upon. This practice can be as simple as a threeminute breath meditation or can expand to a fifteen-minute body scan, based on the context and participants' needs.

\section{Sharing Community Agreements and Gathering Consent: Door - "Welcome"/ "Enter"}

The practice of community agreements is adapted from the Hass Institutes' Bridging and Breaking seminars and the Robert Wood Johnson Foundation Executive Nurse Fellowship Program. This portion of the model asks the participants to agree to a set of specific community agreements. Eliciting group buy-in of the community agreements involves repeating and visually showing these agreements in the room (on a white board, etc.) and all participants verbally agreeing to these statements by vocalizing "yes" if they agree. The community agreements used at the Center for Mindfulness, Compassion, and Resilience events are (1) allow yourself to deeply listen; (2) recognize that we all carry wisdom; (3) seek to understand first, then seek to be understood; and (4) choose a love-based response before a fearful one.

The verbal agreements have two purposes. First, they invite the participants to broaden their perspective by remaining curious for what is to come. Second, verbally agreeing to the group agreements enables the participants see the others within the group committing to community, i.e., each participant is in accord with the others. If a person does not agree or would like to change or amend an agreement, this is also welcomed. The agreement space is the 
time for people in the room who might be hesitant to voice their opinions within the room. Participants who state opposing opinions and then witness agreement of the other participants gain a sense of belonging in the group, creating a tighter container for community experiences. This step is depicted as a door because by operating on community agreements, the facilitator gives each participant a chance to "enter" the container that is being created on their own accord.

\section{Giving Thanks to Communities that Have Deep Importance, but Who are Frequently not Acknowledged: Window - "Out-group/In-group"}

For this step, the practitioner chooses which communities should be acknowledged based on the population they are working with. The standard communities for higher education include Indigenous people and lands on which the institution is residing; people of enslavement and indentured servitude that built buildings, institutions, and economies of that country/space; and all the ancestors of the participants. This step is depicted by the window. We do not always acknowledge the significance of a window in our homes. Although it may not structurally imperative, a window is what brings light into our homes and allows us to see a different environment than what we have inside our homes. Having a window gives perspective. Even if the home is locked, being able to see within the house from the outside allows an onlooker access to the inside of the home. Without a window, residents would not have access to see what is outside and invite the light in. Translating this to the theory, by acknowledging these overlooked or unacknowledged populations, the practitioner hopes to give participants in the room who might identify with these groups a way of seeing themselves as a part of the important structure and to give individuals who have already been comfortable within the group/structure (or container) a new perspective.

\section{Laying Groundwork for Community by Narrative Sharing: Roof - "Sealing the Container"}

Narrative and story sharing support in-group experiences are used by individuals within the community to communicate their relationship to the in-group of which they are members. Narrative sharing has been shown to facilitate kinship and describe an individual's relationship within a community (Goodson \& Gill, 2011; Srinivasan, 2002; Hinchman \& Hinchman, 1997). This step is the final piece of creating a brave container for shared space with the Equitable Mindfulness model for the conversation topic. By introducing the conversation topic through the narrative of a person who is reflective of that specific population, the community members have a prototype of how they can and should engage. This narrative should be 5 - 10 minutes in which the individual shares their experiences as related to the topic or difficult conversation. This step is depicted as the roof because the planned topic narrative "seals" the container and gives a framework for how to be brave in sharing their experiences within the container. By this step, participants should begin to feel a sense of community. The facilitator's job is to hold space for the conversations and experiences and use the intentional pause of mindfulness to refocus participants when the conversation wanders, or when emotions are high.

Finally, while the facilitator holds space for the discussion, all participants are acknowledged and referred to as "knowledge sharers." While the facilitator may have specific academic, personal, or professional experience on the topic being discussed, the authors appreciate that all participants — in fact, all people — have deep wisdom to be shared with the world. Recognizing this wisdom and intentionally naming all participants "knowledge sharers" helps flatten hierarchical barriers to genuine communication and break down unnecessary privilege structures. 


\section{SUMMARY}

Equitable Mindfulness is being explored as a way to create a more universal lens and broader conversations around our understanding of secular mindfulness. It is also being investigated as a means to support curriculum by providing resources to elicit shared spaces within a community through the construction of brave containers. These concepts have been shared through different platforms and within various sectors. Equitable Mindfulness was used in conjunction with an expert opinion on National Public Radio (NPR) to detail the possible risks of incorporating mindfulness in schools without including a lens of equity (Kamenetz \& Knight, 2020). Locally, the framework is being used at conferences, events, workshops, gatherings, and classrooms to highlight the intersections of inclusive practices and mindfulness. The background, concept, and model all work to bridge communities by breaking perceived societal and cultural barriers to promote open conversation about difficult topics within curricula (racism, xenophobia, sexism, implicit bias, diversity and inclusion, etc.) and recognizing the inherent benefit of mindfulness for all people.

In the current context, Equitable Mindfulness has become integral in teaching settings where difficult conversations are being discussed. In a brave container, students, participants, educators and practitioners discuss anti-oppressive and anti-racist themes with foundations of compassion and resilience. The authors and researchers hope that this content is translatable and foundational to the work being done globally.

\section{CONCLUSION}

Equitable Mindfulness represents an opportunity for higher education and other sectors to use intention and cocreation to provide containers and that invite mindfulness and contemplative experiences for all. In this way, mindfulness moves beyond a focus on the individual to include a more expansive societal perspective and works to remove the perceived barriers to practice in the western secular and medical fields. More work is needed to explore how Equitable Mindfulness will inform and inspire existing and emerging programs, workshops, curricula, and organizations. As Equitable Mindfulness works to bridge communities and break barriers to exclusive spaces, the researchers encourage academics and practitioners from all sectors that serve higher education to consider how Equitable Mindfulness can strengthen and amplify their own service work. Mindfulness programs exist within localized environments, often reaching people with similar demographic identifiers (Cullen, 2011). Equitable Mindfulness can expand the reach of mindfulness programs increase inclusivity, reach a wider audience, and, ultimately, create a more equitable society. 


\section{REFERENCES}

Arizona State University Charter (n.d.). In Arizona State University. Retrieved from https://www.asu.edu/about/charter-mission-and-values

Arizona State University fall enrollment numbers (n.d.). In asu.edu. Retrieved from https://www.asu.edu/about/enrollment

Bautista, T. G., Cash, T., Dunis, J., Smith, P. G., Young, M., Meyerhoefer, T., \& Pipe, T. B. (2019). Equitable Mindfulness for social changes: A mixed methods analysis. Poster presented at the American Public Health Association Conference, Philadelphia, PA.

Berila, B. (2015). Integrating mindfulness into anti-oppression pedagogy: Social justice in higher education. Routledge. https://doi.org/10.4324/9781315721033

Bohecker, L., \& Doughty Horn, E. A. (2016). Increasing students' empathy and counseling self-efficacy through a mindfulness experiential small group. Journal for Specialists in Group Work, 41, 312-333. https://doi.org/10.1080/01933922.2016.1232322

Cannon, J. (2016). Education as the practice of freedom: A social justice proposal for mindfulness educators. In Handbook of Mindfulness (pp. 397-409). Springer, Cham. https://doi.org/10.1007/978-3-319-44019-4_26

Carmody, J., \& Baer, R. A. (2008). Relationships between mindfulness practice and levels of mindfulness, medical and psychological symptoms and well-being in a mindfulness-based stress reduction program. Journal of Behavioral Medicine, 31(1), 23-33. https://doi.org/10.1007/s10865-007-9130-7

Cash, T. (n.d.). Equitable Mindfulness Initiative. Retrieved from https://mindfulnesscenter.asu.edu/equitablemindfulness-initiative

Cullen, M. (2011). Mindfulness-based interventions: An emerging phenomenon. Mindfulness, 2, 186-193 https://doi.org/10.1007/s12671-011-0058-1

Forbes, D. (2016). Modes of mindfulness: Prophetic critique and integral emergence. Mindfulness, 7(6), 1256-1270. https://doi.org/10.1007/s12671-016-0552-6

Fulton, P. R., \& Siegel, R. D. (2013). Buddhist and western psychology: Seeking common ground.

García-Campayo, J., Demarzo, M., Shonin, E., \& Van Gordon, W. (2017). How do cultural factors influence the teaching and practice of mindfulness and compassion in Latin countries? Frontiers in Psychology, 8, 1161. https://doi.org/10.3389/ fpsyg.2017.01161

Goodman, A. (2019). Conference to explore the power of mindfulness for social change. In ASU Now. Retrieved from https://asunow.asu.edu/20190103-conference-explore-power-mindfulness-social-change

Goodson, I., \& Gill, S. (2011). Narrative pedagogy: Life history and learning (Vol. 386). Peter Lang.

Gonzalez, T. (2017). Root to rise: Mindful lawyering for social justice. NYU Rev. L. \& Soc. Change, 41, 91.

Grabovac, A. D., Lau, M. A., \& Willett, B. R. (2011). Mechanisms of mindfulness: A Buddhist psychological model. Mindfulness, 2(3), 154-166. https://doi.org/10.1007/s12671-011-0054-5 
Grossman, P., Niemann, L., Schmidt, S., \& Walach, H. (2004). Mindfulness-based stress reduction and health benefits: A meta-analysis. Journal of Psychosomatic Research, 57(1), 35-43.

https://doi.org/10.1111/j.2042-7166.2003.tb04008.x

Gueci, N. \& Cash, T. (2020, March). The Expansive Model of Mindfulness. LinkedIn.

https://www.linkedin.com/pulse/expansive-model-mindfulness-nika-gueci-tiara-cash-nika-gueci/

Hall, K. E. (2009). Anxiety and counseling self-efficacy among counseling students: The moderating role of mindfulness and alexithymia (Doctoral dissertation). Retrieved from ProQuest Dissertations and Theses Global database. (UMI No. 3356126)

Hanson, K., McLeod, C., \& Ross, M. (2017). Mindfulness and Nature. A mindful teaching community: Possibilities for teacher professional learning, 89.

Harris, A. P. (2011). Toward lawyering as peacemaking: A seminar on mindfulness, morality, and professional identity. Journal of Legal Education, 61, 647.

Hass Institute, Whole Story, \& Studio Pathways. (2019). PDF.

Hawley, L. L., Schwartz, D., Bieling, P. J., Irving, J., Corcoran, K., Farb, N. A., ... Segal, Z.V. (2014). Mindfulness practice, rumination and clinical outcome in mindfulness-based treatment. Cognitive Therapy and Research, 38(1), 1-9. https://doi.org/10.1007/s10608-013-9586-4

Henrich, J., Heine, S., \& Norenzayan, A. (2010). The weirdest people in the world? Behavioral and Brain Sciences, $33(2-$ 3), 61-83. https://doi.org/10.1017/S0140525X0999152X

Hick, S., \& Furlotte, C. (2009). Mindfulness and social justice approaches: Bridging the mind and society in social work practice. Canadian Social Work Review/ Revue Canadienne De Service Social, 26(1), 5-24.

Hinchman, L. P., \& Hinchman, S. (Eds.). (1997). Memory, identity, community: The idea of narrative in the buman sciences. Suny Press.

Kabat-Zinn, J. (2012). Mindfulness for beginners: Reclaiming the present moment—and your life. Sounds True.

Kabat-Zinn, J. (1990). Full catastrophe living: Using the wisdom of your body and mind to face stress, pain, and illness. New York, NY: Bantam Doubleday Dell.

Kamenetz, A., \& Knight, M. (2020). Schools are embracing mindfulness, but practice doesn't always make perfect. Retrieved from https://www.npr.org

Kerr, C. E., Sacchet, M. D., Lazar, S. W., Moore, C. I., \& Jones, S. R. (2013). Mindfulness starts with the body: Somatosensory attention and top-down modulation of cortical alpha rhythms in mindfulness meditation. Frontiers in Human Neuroscience, 7. https:/ / doi.org/10.3389/ fnhum.2013.00012

Krygier, J. R., Heathers, J. A., Shahrestani, S., Abbott, M., Gross, J. J., \& Kemp, A. H. (2013). Mindfulness meditation, well-being, and heart rate variability: A preliminary investigation into the impact of intensive Vipassana meditation. International Journal of Psychophysiology, 89(3), 305-313. https://doi.org/10.1016/j.ijpsycho.2013.06.017

Le, T. N., \& Shim, P. (2014). Mindfulness and the Aloha response. Journal of Indigenous Social Development, 3(2). 
Magee R.V. (2016) Community-Engaged Mindfulness and Social Justice: An Inquiry and Call to Action. In: Purser R., Forbes D., Burke A. (eds) Handbook of Mindfulness. Mindfulness in Behavioral Health. Springer, Cham. https://doi.org/10.1007/978-3-319-44019-4_28

Mindfulness comes to ASU (n.d.). In Arizona State University Center: Mindfulness, Compassion and Resilience. Retrieved from https://mindfulnesscenter.asu.edu/

Montero-Marin, J., Tops, M., Manzanera, R., Piva Demarzo, M., Álvarez de Mon, M., \& García Campayo, J. (2015). Mindfulness, resilience, and burnout subtypes in primary care physicians: The possible mediating role of positive and negative affect. Frontiers in Psychology, 6, 1895. https://doi.org/10.3389/fpsyg.2015.01895

Nussbaum, B. (2013). African culture and Ubuntu reflections of a South African in America. World Business Academy, 17(1), 1-12.

Olendzki, A. (2005). The Roots of Mindfulness. In C. K. Germer, R. D. Siegel, \& P. R. Fulton (Eds.), Mindfulness and psychotherapy (p. 241-261). Guilford Press.

Phillips, F. B. (1990). NTU psychotherapy: An Afrocentric approach. Journal of Black Psychology, 17, 55-74. https://doi.org/10.1177/00957984900171005

Pipe, T. B., \& Bortz, J. J. (2009). Mindful leadership as healing practice: Nurturing self to serve others. International Journal for Human Caring, 13, 34-38. https:/ / doi.org/10.20467/1091-5710.13.2.34

Pipe, T. B., Bortz, J. J., Dueck, A., Pendergast, D., Buchda, V., \& Summers, J. (2009). Nurse leader mindfulness program for stress management: A randomized controlled trial. Journal of Nursing Administration, 39, $130-137$. https://doi.org/10.1097/NNA.0b013e31819894a0

Robins, C. J. (2002). Zen principles and mindfulness practice in dialectical behavior therapy. Cognitive and Behavioral Practice, 9(1), 50-57. https://doi.org/10.1016/S1077-7229(02)80040-2

Rogers, H. B. (2016). The mindful twenty-something. Oakland, CA: New Harbinger.

Srinivasan, R. (2002). Village Voice: Expressing narrative through community-designed ontologies (Doctoral dissertation, Massachusetts Institute of Technology).

Woods-Giscombé, C. L., \& Gaylord, S. A. (2014). The cultural relevance of mindfulness meditation as a health intervention for African Americans: Implications for reducing stress-related health disparities. Journal of Holistic Nursing, 32(3), 147-160. https:/ / doi.org/10.1177/0898010113519010

Wong, Y. L. R. (2004). Knowing through discomfort: A mindfulness-based critical social work pedagogy. Critical Social Work, 5(1), 1-19 
Address Author correspondence to:

Tiara Cash, MS

Culture and Equity Specialist

Arizona State University

Edson College of Nursing and Health Innovation

Center for Mindfulness, Compassion, and Resilience

455 N. 3rd Street - Suite 350

Phoenix, AZ 85004

Tiara.cash@asu.edu

Nika Gueci, EdD, MA

Executive Director, University Initiatives

Arizona State University

Edson College of Nursing and Health Innovation

Center for Mindfulness, Compassion, and Resilience

455 N. 3rd Street - Suite 350

Phoenix, AZ 85004

Nika@asu.edu

Teri Pipe, PhD, RN

ASU Chief Well-Being Officer

Founding Director, ASU Center for Mindfulness, Compassion and Resilience

Professor and Dean Emerita

Arizona State University

Edson College of Nursing and Health Innovation

500 N. Third Street

Phoenix, AZ 85004

Teri.Pipe@asu.edu

\section{Author's Note and Acknowledgements}

The authors would like to thank the participants of the Equitable Mindfulness Gatherings and our $2^{\text {nd }}$ Annual Conference - Water \& Stone: The Power of Mindfulness for Social Change. Additionally, the authors would like to thank Amanda Applegate for her contributions to editing the manuscript. And finally, we would like to humbly thank all of the powerful teachers, practitioners, and meditators who have paved the way for this work to be possible. 